\title{
Aprendizaje de la metodología Lean mediante la simulación de un proceso de embotelladora
}

\author{
Oscar Trull-Domínguez ${ }^{a}$, Ángel Peiró-Signes ${ }^{b}$, Marival Segarra-Oña ${ }^{c}$ y Alba Ladera \\ Pescador $^{\mathrm{d}}$ \\ ${ }^{a}$ Departamento de Estadística e Investigación Operativa Aplicada y Calidad (Universitat Politècnica de \\ València, otrull@eio.upv.es), bDepartamento de Organización de Empresas (Universitat Politècnica de \\ València, anpeisig@omp.upv.es), 'Departamento de Organización de Empresas (Universitat \\ Politècnica de València, maseo@omp.upv.es) y s'Autoliv BKI S.A.
}

\begin{abstract}
This paper describes the development of an experiential learning activity to instruct the students in the Lean and Lean 6 sigma techniques throught the simulation of a bottler company.

The processes for the planning, implementation and development of the activity are described, as well as the results of our experience in its realization. These results show how the use of experiential activities improves motivation and student participation in the activity, which strengthens the learning of concepts and skills.
\end{abstract}

Keywords: Experiential Learning Activities, Control plans, Quality Management, transversal competences

\section{Resumen}

Este articulo describe el desarrollo de una actividad de aprendizaje experiencial para la formación de los alumnos en técnicas de Lean y Lean 6 sigma mediante la simulación de una embotelladora.

Se describen los procesos para la planificación, implementación y desarrollo de la actividad, así como se muestran los resultados de nuestra experiencia en su realización. Estos resultados muestran cómo la utilización de las actividades experienciales mejoran la motivación y la participación del estudiante en la actividad, lo que fortalece el aprendizaje de los conceptos y habilidades.

Palabras clave: Actividad de Aprendizaje experiencial, Planes de control, Gestión de Calidad, competencias transversales. 


\section{Introducción}

La enseñanza de los procesos Lean no puede abarcarse de forma tradicional mediante una lección magistral que los alumnos escuchan, comprenden e interiorizan. En general, los alumnos no gozan de experiencia laboral, y no pueden comprender la problemática existente en un proceso. Es por ello que muchos autores han intentado demostrar la conveniencia de la enseñanza de las metodologías Lean mediante el aprendizaje basado en problemas (PBL). Johnson et al. (2003) inciden en la necesidad de aprender estas técnicas mediante la experiencia. Verma et al. (2003) analizaron la situación para la enseñanza de este tipo de técnicas en la industria naval, y determinaron que a pesar de la conveniencia de esta práctica, sigue utilizándose únicamente una de estas actividades para la enseñanza, el Beer Game. Duskovska-Popovska et al. (2008) analizaron los retos a los que se enfrenta esta metodología de enseñanza. Por otro lado, Badurdeen et al. (2010) analizaron los juegos y simulaciones más comúnmente utilizados. Destacan como desde el primer Beer Game (1963) hasta 1994 no ha habido prácticamente trabajos publicados, y como, a partir de ese momento, surge una explosión de trabajos. En la línea de este trabajo Ellis et al. (2014) utilizan una cadena de montaje de un avión para aplicar la enseñanza del Lean Seis Sigma.

\section{Objetivos}

El objetivo de este artículo es desarrollar una Actividad de Aprendizaje Experiencial para la enseñanza de técnicas Lean y 6 sigma. Utilizando una simulación de un proceso de una embotelladora de líquidos, incluida la distribución, los alumnos aprenderán sobre la necesidad de eliminar despilfarros en el proceso, y posteriormente aplicar técnicas específicas de las metodología Lean para mejorar la productividad.

La utilización de una actividad experiencial consigue, además de lograr los objetivos de aprendizaje conceptuales, la formación en competencias transversales como: trabajo en equipo, liderazgo, organización y planificación, entre otros.

\section{Desarrollo de la innovación}

LaForge y Busing (1998) establecieron como organizar una actividad experiencial de aprendizaje de forma efectiva. Para ello, establecieron cuatro fases bien diferenciadas: La planificación, la introducción a la actividad, la ejecución, y la síntesis o reflexión sobre la misma.

Durante la fase de planificación, el profesor procura diseñar una actividad que simule una situación lo más cercana a la realidad, salvando las limitaciones en materia de recursos, tiempo, etc. Es importante considerar los conocimientos previos del alumnado para establecer los objetivos y el proceso de desarrollo (Halpern y Hakel, 2003). Esta fase es fundamental a la hora de decidir el desarrollo de la actividad. Debe preverse las posibles dudas, reflexiones, $\mathrm{y}$ adversidades a las que deben enfrentarse los alumnos durante el transcurso de la actividad. 
Esta parte es fundamental para mantener el nivel de atención y motivación durante el desarrollo de la actividad (Kolb, 2014; Wheeler y McLeod, 2002).

La fase de introducción permite al profesor poner en situación a los alumnos, realizar la toma de contacto con la actividad, y, sobretodo, establecer las reglas de trabajo para la actividad.

La fase de desarrollo exige la mayor atención del profesor frente al resto de fases. El profesor debe facilitar la actividad de los alumnos durante la experiencia para orientarlos hacia los objetivos previstos. Una buena planificación además ayuda a conseguir que la actividad fluya de una manera más natural y en un ambiente más relajado. La experiencia adquirida en la ejecución de la actividad permitirá al profesor sobreponerse a situaciones anómalas que puedan surgir durante su desarrollo. Es importante que el alumno durante esta enfocado e inmerso en la realización de las tareas propuetas.

Por último, es necesario un proceso de reflexión y discusión por parte de los alumnos sobre lo ocurrido durante la actividad. Esta reflexión dirigida por el profesor es la base para el aprendizaje del alumno, donde se relacionan los conceptos previamente aprendidos con la experiencia de la actividad para construir nuevos aprendizajes. En esta fase el profesor también puede obtener información la valoración de los alumnos sobre la actividad, tanto en lo referente a la adquisición de conceptos y habilidades, como en lo relativo al desarrollo de la actividad. Esta información puede utilizarse en una análisis de lecciones aprendidas para evolucionar y mejorar la actividad, evitando errores que pudieron surgir en el pasado.

\subsection{Planificación}

La respuesta del alumnado está directamente relacionada con la planificación de las tareas. La motivación y el ritmo de la actividad debe estar controlado en todo momento, de modo que la actividad no diverja en un simple juego, ni que frustre las expectativas de los alumnos.

La planificación atiende a las peculiaridades de la formación, analizando los espacios, los recursos disponibles, así como los necesarios para llevar a cabo la actividad. Debido a que se requiere de instrumentación específica, es necesario disponer de cronómetros $\mathrm{y}$, en la medida de lo posible, dispositivos de grabación. En la Tabla 1 se muestra un resumen de los materiales necesarios.

Uno de los objetivos transversales clave es el trabajo en equipo. Los alumnos se deben dividir en grupos y cohabitar dentro del mismo proceso. Esto permite el desarrollo cognitivo de habilidades específicas, como liderazgo y la comunicación, entre otras. La resolución de problemas es una de las habilidades clave trabajadas durante la ejecución de la experiencia: los alumnos, trabajando en grupo, deben detectar y resolver los problemas de producción existentes, con la guía del profesor.

Tabla 1. Material de trabajo Lean necesario para la actividad. 


\begin{tabular}{lcc}
\hline \multicolumn{1}{c}{ Material } & Objeto & Obligatoriedad \\
\hline Plantilla asignación de roles & Establecer Rol & Obligatorio \\
Operación estándar & Tareas, Kaizen & Obligatorio \\
Tijeras, Grapadora, & Producción & Obligatorio \\
Lápices, rotuladores, papel & & \\
Plantillas de producción & Producción & $\begin{array}{c}\text { Opcional. Mejora los } \\
\text { tiempos de trabajo. }\end{array}$ \\
Plantilla de verificación. & Calidad & $\begin{array}{c}\text { Opcional. Debería formar } \\
\text { parte del proceso. }\end{array}$ \\
Cronómetros & Productividad & Obligatorio \\
Dispositivo de grabación & Productividad & Opcional \\
\hline
\end{tabular}

\subsection{Introducción}

La fase inicial con los alumnos supone una introducción al problema a tratar. Los alumnos son desconocedores de estas técnicas, además muchos de ellos no están concienciados con la eficiencia en el trabajo. Es necesario aportar ejemplos de su día a día en el que existan ineficiencias manifiestas. Esta situación debe extrapolarse a los procesos industriales o de servicios, y en general, a cualquier tipo de proceso.

La actividad consiste en la simulación de una embotelladora. Sugerimos entablar una pequeña conversación con los alumnos, de no más de dos minutos, con la que determinar qué tipo de líquido va a ser embotellado. A partir de ahí, se analizan los procesos necesarios, y se guía a los alumnos hacia un proceso lineal. Además, para conseguir una actividad más motivadora, el total de alumnos del aula se divide en, al menos, dos grupos o más, manteniendo un número de integrantes por grupo del mínimo necesario para realizar la actividad, que son 6 .

Para garantizar un correcto funcionamiento de la actividad, es necesario una explicación profunda de las reglas de trabajo durante la sesión. El uso de documentación con compromiso, que incluya la firma del alumno, consigue elevar de forma significativa la implicación del alumno en la actividad.

\subsection{Actividad}

La fase inicial consiste en asignar los roles de trabajo para cada uno de los integrantes de los grupos y en distribuir el aula para poder llevar a cabo el ejercicio. En la Fig. 1 se muestra la asignación de roles, en la que cada integrante del grupo se compromete a realizar las acciones de modo eficiente y buscando la máxima productividad. Los roles se asignan para cada tarea y un operario se encarga de la supervisión. Existe rol de control de calidad opcional para el 
caso de que la distribución por número de integrantes en distintos grupos lo requiera. En el caso de no ser así, el operador 4 realizará las tareas de control de calidad.

La disposición inicial de las mesas es importante. El profesor no debe responder con claridad a los motivos de la distribución, simplemente aludiendo a que la disposición "habitual" del aula se conforma de acuerdo a la Fig. 2.

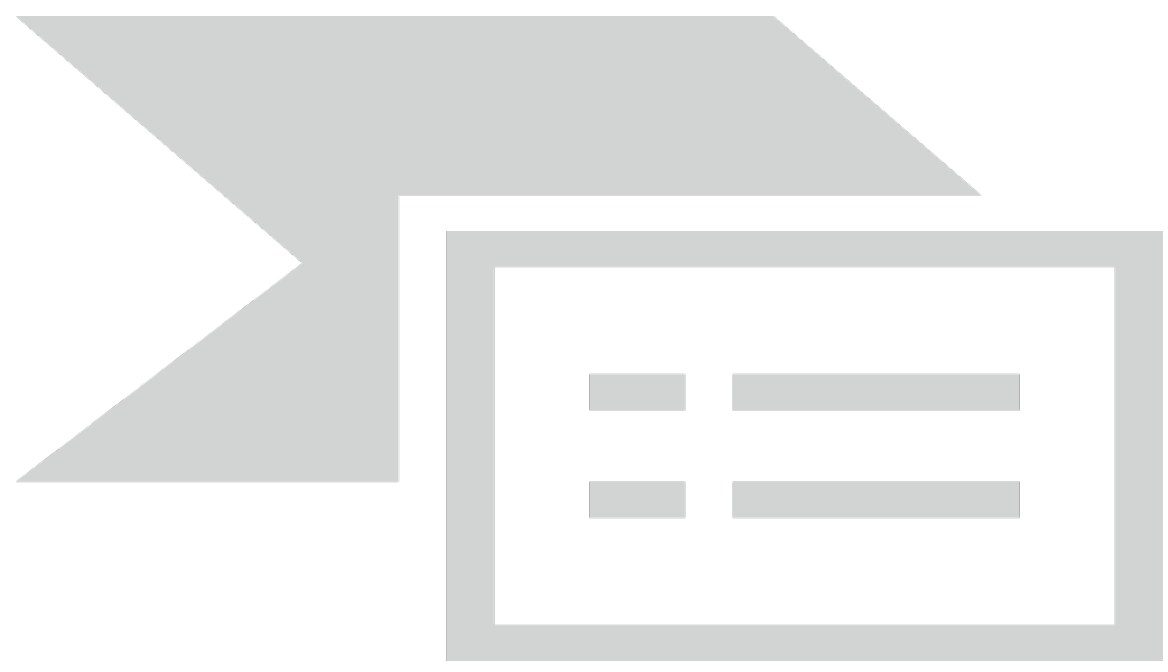

Fig. 1 Plantilla de asignación de roles en la actividad.

En un momento inicial, se describen los procesos a realizar, y la organización de los mismos, tal y como se indica en la Fig. 3. Es importante que los alumnos dispongan de las tareas que deben realizar en cada rol. Para ello se les proporciona una hoja de operación estándar donde se les indica cada uno de los pasos que deben realizar.

El proceso de trabajo se realiza durante unos minutos. Es necesario que se produzca, al menos, un par de botellas. No importa el proceso que hayan seguido, si bien es importante, tras estos minutos, recabar cierta información. Es importante determinar el tiempo requerido para cada operación, así como también, en caso de ser posible, disponer de una grabación del proceso para posteriormente ser visualizada.

Se plantea un debate con el proceso de producción. Por norma general, los alumnos escogen de entre dos modos de producción: lineal o por lotes. En cualquier caso, hay que revisar la cantidad de material realizado, y la cantidad de material disponible en stock intermedio. Si el tiempo disponible para la actividad es suficiente, debe cambiarse el método de producción al alternativo, con el fin de comprobar que ambos métodos producen grandes ineficiencias en la situación actual. En la Fig. 4 se muestra un ejemplo de la situación posible durante esta realización. 


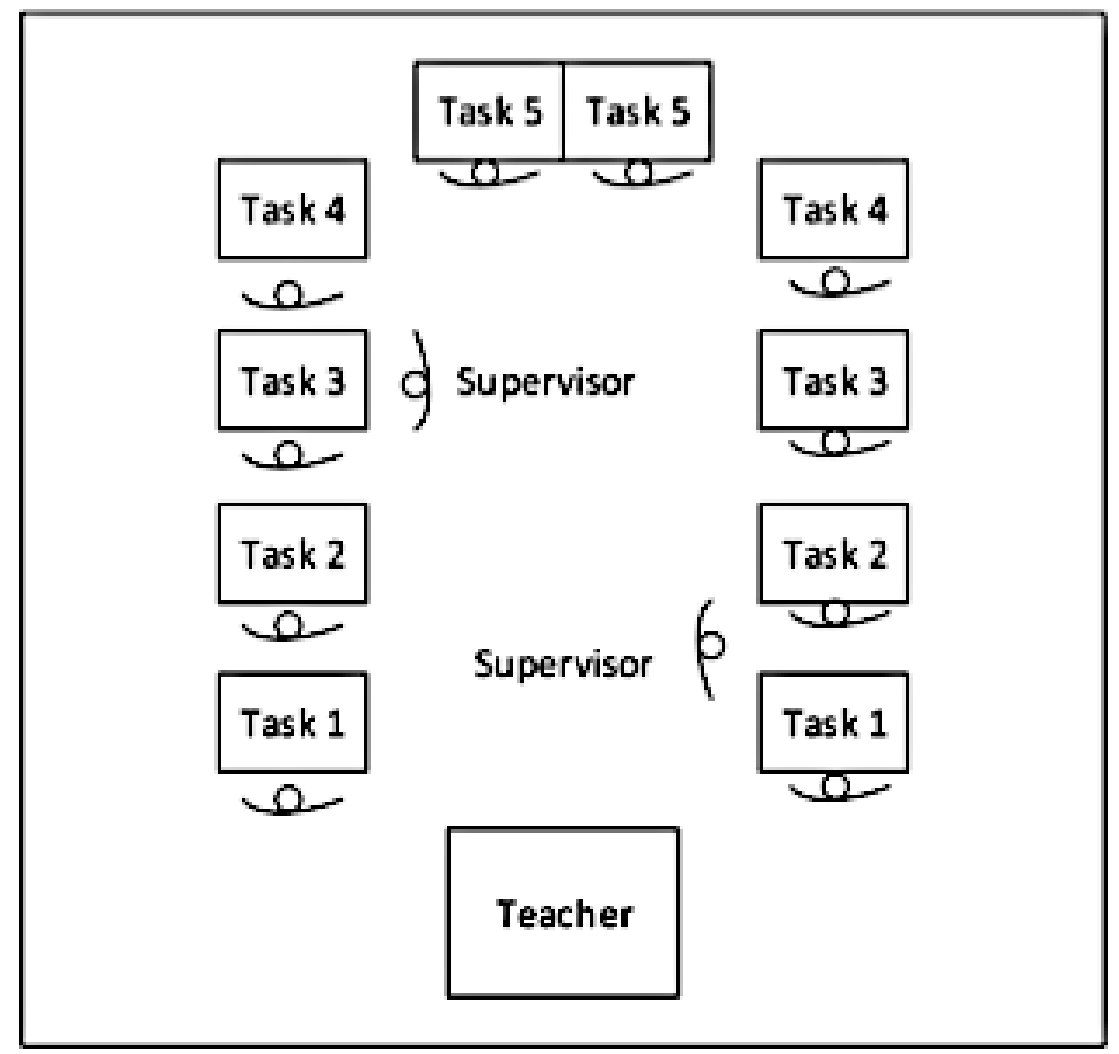

Fig. 2 Disposición inicial de los procesos en el aula.

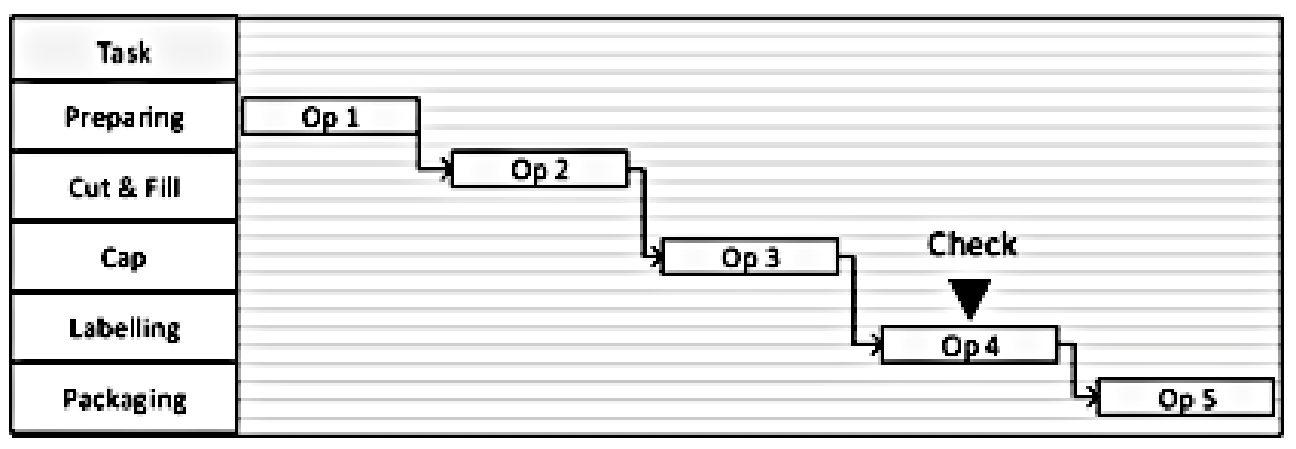

Fig. 3 Gantt simplificado de la organización de las tareas a realizar por cada integrante del grupo.

A partir de este momento es necesario realizar una primera reflexión y es donde el profesor va a dirigir a los alumnos hacia la enseñanza en Lean Manufacturing.

Se analizan los tiempos en cada proceso, y se visualizan las grabaciones, en caso de disponerlas. El tema económico adquiere especial relevancia, de modo que se cuantifica económicamente todas las acciones, y prestando especial atención a los stocks intermedios y 
desechos, que no aportan liquidez económica al grupo. Se ponen en marcha los diferentes principios del Lean (ver Tabla 2) para mejorar el proceso.

Tabla 2. Principios del Lean desarrollados en la actividad.

\begin{tabular}{lc}
\hline \multicolumn{1}{c}{ Concepto } & Herramienta \\
\hline $5 \mathrm{~S}$ & Videos, Stocks \\
Control Visual & Plantillas \\
Trabajo Standard & Hojas de trabajo estándar \\
Takt time & Cronómetros, hoja de \\
& cálculo \\
Trabajo balanceado & Hoja de cálculo, videos \\
Just In time & Stock, eliminación del \\
& sobrante \\
MUDA & Estandarización de trabajos \\
\hline
\end{tabular}

El profesor guía a los alumnos a resolver problemas poco a poco, mediante la filosofía del Kaizen y utilizando los distintas herramienta Lean, con el fin de que los procesos mejoren. Esta mejora se debe realizar en varias etapas, siendo una de ellas fundamental la disposición del aula. La filosofía de trabajo debe cambiar un sistema push a un sistema pull. El profesor guía a los alumnos permitiendo que los alumnos desarrollen ideas creativas que mejoren el proceso. El resultado final de este proceso lleva generalmente a una disposición de los distintos puesto de trabajo como la mostrada en la Figura 4, con la determinación stocks intermedios y kanban, la utilización de códigos de colores, el establecimiento y delimitación de zonas de trabajo, etc.

No todos los alumnos e integrantes de cada grupo se desenvuelven de la misma forma aplicando las técnicas Lean en el aula. No existe un objetivo principal de reducir los tiempos de producción por debajo de un nivel establecido. El profesor debe concienciar a los alumnos de esta situación.

Al finalizar la actividad, se debe hacer un balance económico de la situación obtenida comparando en cada etapa los resultados y mejora con respecto a la situación inicial. Se muestra como ejemplo la Tabla 3. En ella aparece la cantidad de alumnos trabajando en la producción, así como los stocks intermedios y el coste real de la producción y ventas. 


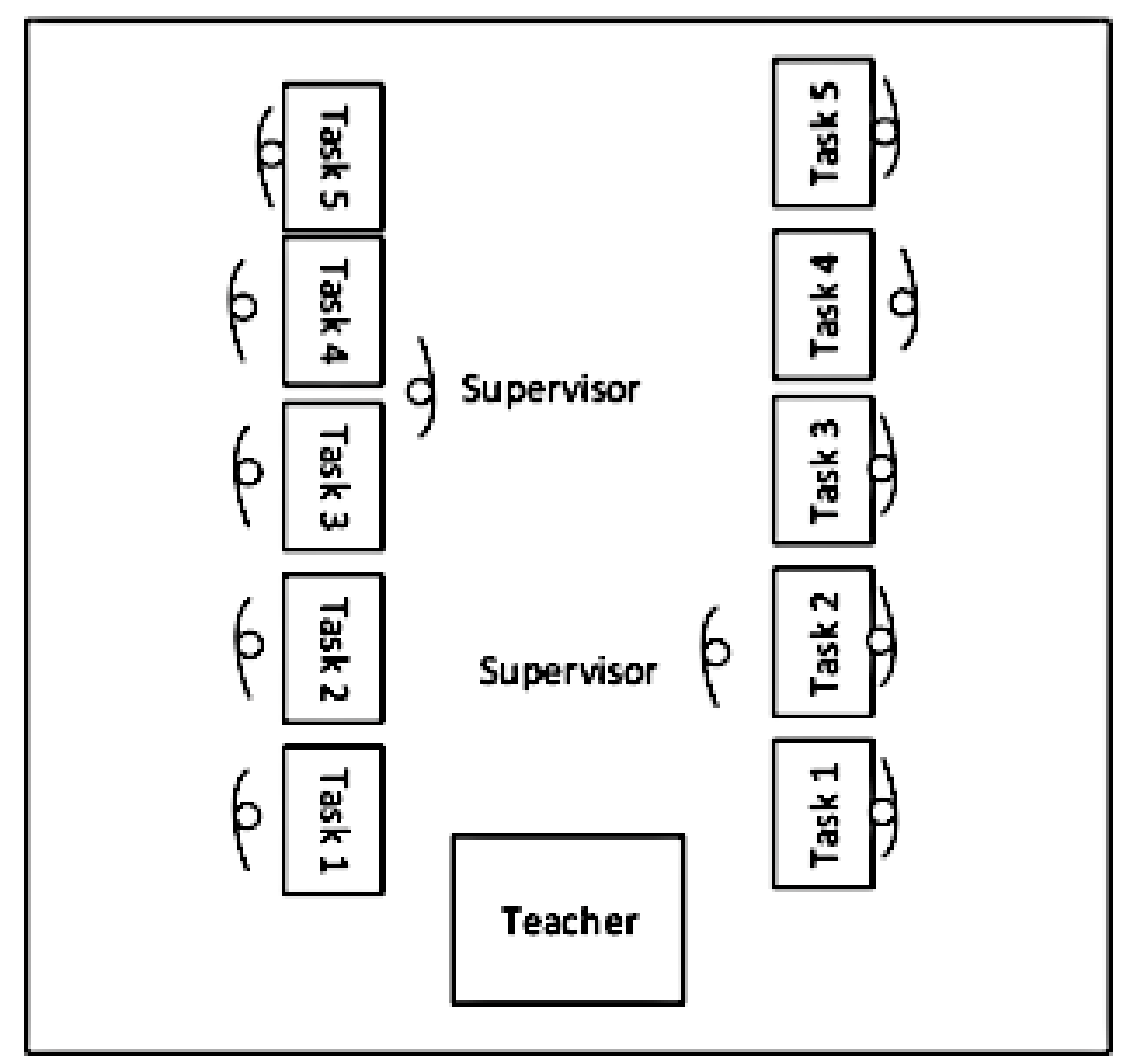

Fig. 4 Disposición final del aula.

Tabla 3. Resultados obtenidos en la producción.

\begin{tabular}{lccc|ccc} 
& \multicolumn{3}{c}{ Factory A } & \multicolumn{3}{c}{ Factory B } \\
\cline { 2 - 7 } & Round & Round & Round & Round & Round & Round \\
& $\mathbf{1}$ & $\mathbf{2}$ & $\mathbf{3}$ & $\mathbf{1}$ & $\mathbf{2}$ & $\mathbf{3}$ \\
\hline OPERATORS & 8 & 7 & 6 & 8 & 7 & 6 \\
PARTS IN WIP & 29 & 21 & 1 & 16 & 15 & 2 \\
SALES & 3 & 10 & 12 & 2 & 4 & 14 \\
LEAD TIME & 10 & 6 & 3 & - & 5 & 2 \\
\hline PROFIT & $\mathbf{- 1 1 5}$ & $\mathbf{- 6 5}$ & $\mathbf{- 3 7}$ & $\mathbf{- 1 0 2}$ & $\mathbf{- 1 0 9}$ & $\mathbf{- 6 0}$
\end{tabular}

Nota: Cada ronda supone una renovación del método de producción, y la última ronda es la definitiva con el proceso optimizado. Los valores negativos indican que todavía podría realizarse mejoras. 


\subsection{Reflexión}

Una vez finalizada la actividad, se produce un periodo de reflexión. Esta fase adquiere una especial relevancia para conseguir la asimilación de la filosofía Lean. Debe haber un diálogo entre el profesor y los alumnos, de modo que los alumnos puedan darse cuenta de lo aprendido así como recibir retroalimentación para la aplicación futura de estas herramientas. Esta fase también permite a los profesores evaluar el grado de comprensión de los principios Lean y obtener información relevante que permita mejorar la experiencia en futuras implementaciones.

Uno de los aspectos importantes para entender la comprensión del alumnado de la actividad se puede conseguir realizando preguntas como: ¿cómo podría aplicarse esta metodología en una situación cotidiana? Al final, se genera un debate en torno a la simpleza de estas técnicas, y a la complicación que encontramos para llevarlas a cabo en el día a día. Nuevas preguntas pueden llevar a los alumnos a entender que es una actitud, más que una herramienta.

Para finalizar, el profesor recapitula todos los puntos a tratar del Lean, y los asocia con los procesos. El objetivo es determinar un nivel de éxito en la consecución de los objetivos establecidos para la actividad.

\section{Resultados}

La evaluación de la actividad es una herramienta necesaria y potente que permite analizar el resultado del proceso de enseñanza-aprendizaje. Se determina desde el punto de vista del alumno la percepción sobre la comprensión de los conocimientos adquiridos. Para tal fin, se distribuye un cuestionario que realiza las preguntas sobre dicha percepción. La valoración del cuestionario utiliza una escala de tipo Likert, reflejando en el 5 un aspecto completamente favorable hacia la actividad, y con un 1 un desacuerdo con la misma.

La Tabla 4 muestra los resultados del cuestionario de evaluación de la actividad en la experiencia realizada.

Estos resultados muestran cómo la comprensión de los conceptos, actitudes y procedimientos es mucho más efectiva realizando la actividad experiencial que realizando una clase magistral única.

Además, la metodología empleada ayuda a la motivación y fomenta la participación del alumnado en la actividad.

El diseño de una experiencia de aprendizaje experiencial parece contribuir a una buena percepción del proceso de aprendizaje y capacitación. Además, los resultados preliminares obtenidos apuntan a valor de este tipo de experiencias para motivar a los estudiantes, trabajar la adquisición de habilidades y técnicas de calidad y una mejor comprensión de su aplicabilidad en entornos industriales. 
Tabla 4. Cuestionario y evaluación de los resultados de la actividad.

\begin{tabular}{l|c}
\hline \hline \multicolumn{1}{c|}{ Preguntas } & Media \\
\hline $\begin{array}{l}\text { Does the activity help you to explain what is a Lean 6 sigma and what is it used } \\
\text { for? }\end{array}$ & 5 \\
\hline $\begin{array}{l}\text { Does the activity help you to explain how a Lean methods can improve a } \\
\text { process? }\end{array}$ & 4.38 \\
\hline $\begin{array}{l}\text { Does the activity help you to discuss the possible problems that can arise in a } \\
\text { process and the actions that can be taken? }\end{array}$ & 4.48 \\
\hline $\begin{array}{l}\text { Does the activity help you to understand how to perform Lean Manufacturing } \\
\text { tecniques and which are the elements to include? }\end{array}$ & 4.65 \\
\hline \begin{tabular}{l} 
Does the activity help you to see the general purpose of using a Lean? \\
\hline $\begin{array}{l}\text { To what extent was the experience better and more motivating than a master } \\
\text { class? }\end{array}$
\end{tabular} & 4.45 \\
\hline $\begin{array}{l}\text { Do you think the activity helped the group to act as a team? } \\
\text { To what extent has communication been important in solving the activity? }\end{array}$ & 4.41 \\
\hline $\begin{array}{l}\text { To what extent have you been able to participate and express your opinions in } \\
\text { the group discussion and in the general discussion? }\end{array}$ & 4.33 \\
\hline $\begin{array}{l}\text { Would you recommend the experience for learning about process improvement } \\
\text { topics? }\end{array}$ & 4.97 \\
\hline \hline
\end{tabular}

\section{Conclusiones}

En este artículo se describe una actividad experiencial para la asimilación de los conceptos fundamentales de la metodología Lean. Se desarrolla una simulación de un proceso de producción de una embotelladora, que permite desarrollar el proceso de enseñanzaaprendizaje mediante una actividad motivadora. Mediante la realización de la actividad, el alumno se encuentra inmerso en un mundo de trabajo donde aprende a utilizar herramientas Lean y es capaz de asimilarlas dentro de una experiencia en lugar de utilizar la metodología convencional de enseñanza.

En el artículo se describen los objetivos, la metodología, y los resultados obtenidos de la realización de la actividad. Además, describe la innovación y cómo los alumnos valoran la realización de la actividad, con su consecuente mejora a nivel académico de la adquisición y puesta en práctica de la metodología Lean.

La motivación conseguida con la simulación de un proceso de la vida real, revierte en una situación ventajosa para el profesor, que introduce estos conceptos de forma sutil y basados en experiencias que viven los propios alumnos, los cuales toman conciencia de la dificultad real de los procesos. Así pues, se concluye que esta metodología mejora las condiciones del aula para la adquisición de estos conceptos de calidad y Lean manufacturing, en contra de las clases puramente magistrales. Claro está, estas clases pueden y deben ser complementadas 
con otro tipo de actividad menos lúdica, pero el principal objetivo de aprendizaje ya estará conseguido.

Las encuestas reflejan que además, el trabajo en equipo sale reforzado, así como las competencias transversales directamente relacionadas con la aplicación del pensamiento práctico y de comprensión e integración.

\section{Agradecimientos}

Este trabajo forma parte del estudio desarrollado por el Grupo de Aprendizaje Experiencial (GAE) creado como EICE en la UPV y por profesores del IES Gonzalo Anaya de Xirivella. Los autores desean agradecer a la UPV por el apoyo a través del PIME 2017-2019 "Adaptación y desarrollo de aprendizajes experienciales al contexto de las asignaturas (II)" y a la Conselleria de Educación, Investigación, Cultura y Deporte de la Generalitat Valenciana a través del Proyecto de innovación e investigación educativa denominado "Adaptación y desarrollo de aprendizajes experienciales al contexto de la formación profesional” (Expediente 938397).

\section{Referencias}

BADURDEEN, F., MARKSBERRY, P., HALL, A., y GREGORY, B. (2010). "Teaching lean manufacturing with simulations and games: A survey and future directions". Simulation \& Gaming, vol. 41, issue 4, p. 465-486.

DUKOVSKA-POPOVSKA, I., HOVE-MADSEN, V., y NIELSEN, K. B. (2008). "Teaching lean thinking through game: Some challenges". 36th European Society for Engineering Education (SEFI) on Quality Assessment, Employability \& Innovation.

ELLIS, S. C., GOLDSBY, T. J., BAILEY, A. M., y OH, J. Y. (2014). "Teaching lean six sigma within a supply chain context: The airplane supply chain simulation". Decision Sciences Journal of Innovative Education, vol. 12, issue 4, p. 287-319.

HALPERN, D. y HAKEL, M. (2003). "Applying the science of learning”. Change, vol. 35, issue 4, p. $36-41$.

JOHNSON, S. A., GERSTENFELD, A., AZELIG, B. R., y MISHRA, S. (2003). "Teaching lean process design using a discovery approach". 2003 ASEE Annual Conference and Exposition: Staying in Tune with Engineering Education, p. 7881-7892.

KOLB, D. (1984). Experiential learning: Experience as the source of learning and development. Englewood Cliffs, NJ: Prentice Hall.

LAFORGE, R. y BUSING, M. (1998). "The use of industrial software to create experiential learning activities in operations management courses". Production and Operations Management, vol. 7, issue 3, p. 325-334.

VERMA, A. K. (2003). "Simulation tools and training programs in lean manufacturing-current status". Report submitted to the National Shipbuilding Research, Advanced Shipbuilding Enterprise Program.

WHEELER, J., y MCLEOD, P. (2002). "Expanding our teaching effectiveness: Understanding our responses to in-the-moment classroom events". Journal of Management Education, vol. 26, issue 6, p. 693-716. 